\title{
CALCULATION OF EMITTANCE EXCHANGE BY WEDGE ABSORBERS WITH ASSOCIATED BEAM EFFECTS FOR INTENSITY FRONTIER EXPERIMENTS *
}

\author{
D. V. Neuffer ${ }^{\#}$, D. Stratakis, Fermilab, Batavia IL 60510, USA
}

\begin{abstract}
Intensity in the g-2 ring at Fermilab is most severely restricted by its small energy acceptance. The g- 2 experiment is exploring the use of wedge absorbers to reduce the energy spread of the $\mu$ beam entering the ring. The absorbers increase the transverse emittance by emittance exchange and multiple scattering. Energy straggling, beam mismatch, and other effects can also reduce the effectiveness, and beam optics can be modified to improve performance. Linear models with corrections are used to estimate the changes in beam acceptances and compared with simulations.
\end{abstract}

\section{INTRODUCTION}

Low energy muon experiments, such as the Fermilabbased mu2e [1,2] and g-2 experiments, [3] have a limited phase space acceptance for useful muons. The g-2 experiment only accepts a momentum spread of $\delta P=$ $\pm 0.1 \%$ around the design momentum of $\sim 3.1 \mathrm{GeV} / \mathrm{c}$. Methods that can increase the number of muons within the momentum acceptance are desirable. Similar or complementary constraints occurred in the exploration of ionization cooling for muons. [4, 5] Wedge absorbers are needed to transform the intrinsic transverse cooling effect to include longitudinal cooling, and introduce exchanges between longitudinal and transverse phase space densities.

A simplified model to describe emittance exchange that can be adapted to small (incremental) and large exchanges was developed, initially for muon cooling experiments. [6,7] In this paper we describe the exchange model and extend it to phase space matching into muon experiments such as g-2. Matching of the wedges and the accompanying beam optics can be optimized to improve the acceptance for these experiments.

L

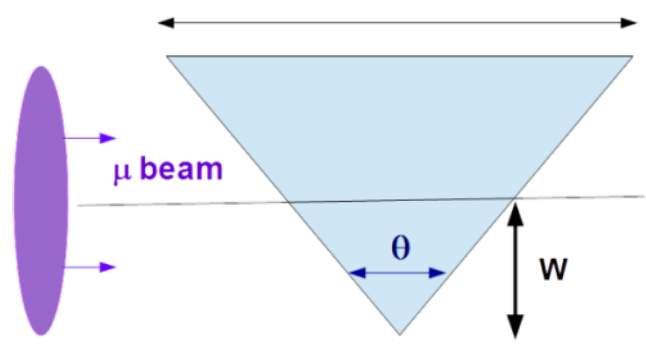

Figure 1: Schematic view of a muon beam passing through a wedge.
The parameters of $\mathrm{g}-2$ and other experiments are discussed and potential uses of wedge absorbers and their adaptation to increase acceptance into the experiments are described. Simulations that test these possibilities are presented and the results are discussed.

\section{WEDGE EFFECTS ON BEAM - FIRST ORDER MODEL}

Figure 1 shows a stylized view of the passage of a beam with dispersion $\eta_{0}$ through a wedge absorber. The wedge is approximated as an object that changes particle momentum offset $\delta=\Delta p / P_{0}$ as a function of $x$, and the wedge is shaped such that that change is linear in $x$. (The change in average momentum $P_{0}$ is ignored in this approximation. Energy straggling and multiple scattering are also ignored.) The rms beam properties entering the wedge are given by the transverse emittance $\varepsilon_{0}$, betatron amplitude $\beta_{0}$, dispersion $\eta_{0}$ and relative momentum width $\delta_{0}$. (To simplify discussion the beam is focussed to a betatron and dispersion waist at the wedge: $\beta_{0}{ }^{\prime}, \eta_{0}{ }^{\prime}=0$. This avoids the complication of changes in $\beta^{\prime}, \eta^{\prime}$ in the wedge.) The wedge is represented by its relative effect on the momentum offsets $\delta$ of particles within the bunch at position $x$ :

$$
\frac{\Delta p}{p}=\delta \rightarrow \delta-\frac{2(d p / d s) \tan (\theta / 2)}{P_{0}} x=\delta-\delta^{\prime} x
$$

$d p / d s$ is the momentum loss rate in the material $(d p / d s=$ $\left.\beta^{-1} d E / d s\right) . \quad 2 x \tan (\theta / 2)$ is the wedge thickness at transverse position $x$ (relative to the central orbit at $x=0$ ), and $\delta^{\prime}=2 d p / d s \tan (\theta / 2) / P_{0}$ to indicate the change of $\delta$ with $x$.

Under these approximations, the initial dispersion and the wedge can be represented as linear transformations in the $x$ - $\delta$ phase space projections and the transformations are phase-space preserving. The dispersion can be represented by the matrix: $\mathbf{M}_{\eta}=\left[\begin{array}{cc}1 & \eta_{0} \\ 0 & 1\end{array}\right]$, since $x \Rightarrow x+\eta_{0} \delta$. The wedge can be represented by the matrix: $\mathbf{M}_{\delta}=\left[\begin{array}{cc}1 & 0 \\ -\delta^{\prime} & 1\end{array}\right]$, obtaining $\mathbf{M}_{\eta \delta}=\left[\begin{array}{cc}1 & \eta_{0} \\ -\delta^{\prime} & 1-\delta^{\prime} \eta_{0}\end{array}\right]$. Writing the $x-\delta$ beam distribution as a phase-space ellipse: $g_{0} x^{2}+b_{0} \delta^{2}=\sigma_{0} \delta_{0}$, and transforming the ellipse by standard betatron function transport techniques obtains

*Work supported by by FRA Associates, LLC under DOE Contract No. DE-AC02-07CH11359.

\#neuffer@fnal.gov 
new coefficients $b_{1}, g_{1}, a_{1}$, which define the new beam parameters[6]. The general expression for this is:

$$
\begin{aligned}
& b_{1}=M_{11}^{2} b_{0}-2 M_{11} M_{12} a_{0}+M_{12}^{2} g_{0} \\
& a_{1}=-M_{11} M_{21} b_{0}+\left(M_{11} M_{22}+M_{12} M_{21}\right) a_{0}-M_{12} M_{22} g_{0} \\
& g_{1}=M_{21}^{2} b_{0}-2 M_{21} M_{22} a_{0}+M_{22}^{2} g_{0}
\end{aligned}
$$

which includes an initial correlation term $a_{0}$. In this formulation, the correlation term $a_{0}$ in the initial ellipse is set to zero. (That term would indicate an initial dispersion, given by $\eta_{I}=-a_{0} / g_{0}$. However, that value would be updated by the dispersion entering the wedge. An initial beam with zero dispersion could then be extrapolated from that beam, and those extrapolated values used as initial parameters; there is no loss of generality in this choice.)

The dispersion plus wedge changes the momentum width to:

$$
\delta_{1}=\sqrt{g_{1} \sigma_{0} \delta_{0}}=\delta_{0}\left[\left(1-\eta_{0} \delta^{\prime}\right)^{2}+\frac{\delta^{\prime 2} \sigma_{0}^{2}}{\delta_{0}^{2}}\right]^{1 / 2} .
$$

The bunch length is unchanged by the wedge. The longitudinal emittance has therefore changed simply by the ratio of energy-widths, which means that the longitudinal emittance has changed by the factor $\delta_{l} / \delta_{0}$. The transverse emittance has changed by the inverse of this factor:

$$
\varepsilon_{1}=\varepsilon_{0}\left[\left(1-\eta_{0} \delta^{\prime}\right)^{2}+\frac{\delta^{\prime 2} \sigma_{0}{ }^{2}}{\delta_{0}{ }^{2}}\right]^{-1 / 2}
$$

The new values of $(\eta, \beta)$ are:

$\eta_{1}=-\frac{a_{1}}{g_{1}}=\frac{\eta_{0}\left(1-\eta_{0} \delta^{\prime}\right)-\delta^{\prime} \frac{\sigma_{0}^{2}}{\delta_{0}{ }^{2}}}{\left(1-\eta_{0} \delta^{\prime}\right)^{2}+\delta^{\prime 2} \frac{\sigma_{0}^{2}}{\delta_{0}{ }^{2}}}$

and $\beta_{1}=\beta_{0}\left[\left(1-\eta_{0} \delta^{\prime}\right)^{2}+\frac{\delta^{\prime 2} \sigma_{0}{ }^{2}}{\delta_{0}{ }^{2}}\right]^{-1 / 2}$.

Note that the change in betatron functions implies that the following optics should be correspondingly matched.

A single wedge exchanges emittance between one transverse dimension and longitudinal; the other transverse plane is unaffected. Serial wedges could be used to balance $x$ and $y$ exchanges, or a more complicated coupled geometry could be developed.

Wedge parameters can be arranged to obtain large exchange factors in a single wedge. In final cooling we wish to reduce transverse emittance at the cost of increased longitudinal emittance. Choosing $\delta^{\prime}=1 / \eta_{0}$, provides a direct exchange of emittance and dispersion beam sizes.

A minimal value of $\delta_{l}$ is obtained by taking

$$
\delta^{\prime}=\frac{1}{\eta_{0}} \frac{\left(\eta_{0} \delta_{0}\right)^{2}}{\left(\eta_{0} \delta_{0}\right)^{2}+\sigma_{x}^{2}}
$$

and $\delta p_{\text {after }}$ is then given by:

$$
\delta p_{\text {after }}=\delta p_{\text {before }}\left(\rightarrow \frac{\sigma_{x}^{2}}{\left(\eta_{0} \delta_{0}\right)^{2}+\sigma_{x}^{2}}\right)^{1 / 2} \text {. }
$$

At this minimum value, the dispersion function after the wedge is canceled to zero.

The choice of a wedge material is dependent on practical considerations. The material should be a relatively low-Z material to minimize mulitiple scattering and must be mechanically compatible with the transport solenoid vacuum pipe. An inexpensive choice is polyethylene $\left(\sim \mathrm{CH}_{2}\right)$, with properties of $\mathrm{Z} / \mathrm{A}=0.57, \rho=0.94, \mathrm{I}_{\mathrm{e}}=57.4$ $\mathrm{eV}, \mathrm{X}_{0}=47.45 \mathrm{~cm}$.[8] This is easily machined, and was actually used to produce a wedge for the MICE experiment. [9] Higher density low-Z materials (Be or $\mathrm{B}_{4} \mathrm{C}$ or $\mathrm{C}$ (diamond)) would provide more compact wedges, which may be needed. $d E / d s$ is $\{2.57,3.86,5.5,8.4\}$ $\mathrm{MeV} / \mathrm{cm}$ for $3.1 \mathrm{GeV} \mu$ 's in spoly, $\mathrm{Be}, \mathrm{B}_{4} \mathrm{C}$, and diamond .

\section{APPLICATION TO G-2}

The g-2 ring has a very small momentum acceptance for $3.1 \mathrm{GeV} / \mathrm{c} \mu$ 's $(\delta \mathrm{P} / \mathrm{P}$ is $\sim 0.1 \%)$. The beam transport into the g-2 ring (which includes the Debuncher ring) has a much larger rms acceptance of $\sim 1.2 \%$. Reduction of that momentum spread before injection into the ring could increase the number of accepted $\mu$ 's. This would require a wedge absorber at a point of the transport with non-zero dispersion.

While a reoptimized lattice with a large dispersion insert might be preferred, we first consider what may be possible by inserting wedges in the current $\mathrm{g}-2$ beam line. Fig. 7 displays betatron functions for the transport into the $\mathrm{g}-2$ ring. The transport to the ring has a horizontal dispersion of $\sim 0.65 \mathrm{~m}$ where $\beta_{\mathrm{x}}=\sim 2 \mathrm{~m}$ and $\beta_{\mathrm{y}}=\sim 7 \mathrm{~m}$, and this might an appropriate location for the wedge, and we choose to explore this as a baseline example.

The (geometric) transverse emittance at this point is $12 \mathrm{~mm}-\mathrm{mrad}$, so $\sigma_{0}{ }^{2}=24 \times 10^{-6} \mathrm{~m}^{2}$, and with $\delta_{0}=0.012$, $\left(\eta_{0} \delta_{0}\right)^{2}=60.8 \times 10^{-6} \mathrm{~m}^{2}$. From eq. 5, the "optimum" $\delta^{\prime}$ is $\sim 1.10 \mathrm{~m}^{-1}$. With this optimum, $\delta_{1}$ would be reduced to $0.64 \%$, with $\varepsilon_{x}$ increased to $22.5 \mathrm{~mm}$-mrad. This would increase beam within the $0.1 \%$ acceptance by $\sim 88 \%$.

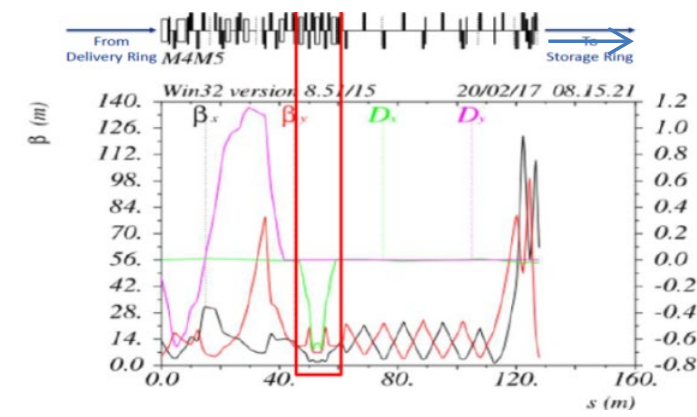

Figure 2. Betatron functions for the transport leading into the g-2 ring.[10, 11] Test wedges are inserted at high dispersion within the highlighted region in simulation.

This optimum $\delta^{\prime}$ corresponds to $\theta \cong 163,154^{\circ}, 142^{\circ}$, $126^{\circ}$ for polyethelene, $\mathrm{Be}, \mathrm{B}_{4} \mathrm{C}$, diamond, respectively. For low-Z materials the wedge would be a somewhat extended object (see fig. 3) because of the large angle. 
This could be split into several shorter wedges of smaller angle, if needed.

The rms $\sigma=\left(\sigma_{x}^{2}+\left(\eta_{0} \delta_{0}\right)^{2}\right)^{1 / 2}$ is $9.2 \mathrm{~mm}$. If the end of the wedge is $\sim 1 \sigma$ from the center of the beam then the energy loss at the center of the beam is $\sim 31.5 \mathrm{MeV}$, which provides a useful guideline in exploring parameters. $(\Delta w=\{12.25,8.2,5.7,3.7\} \mathrm{cm}$ for $\{$ poly, $\mathrm{Be}, \mathrm{B} 4 \mathrm{C}$, diamond $\}$.)

The increase in rms normalized emittance caused by multiple scattering in the absorber can be estimated by

$$
\delta \varepsilon_{N}=\beta_{t} \frac{E_{s}{ }^{2}}{2 \beta^{3} \gamma\left(m c^{2}\right)^{2}} \frac{\Delta w}{L_{R}},
$$

where $E_{s}=13.6 \mathrm{MeV}, \beta_{t}$ is the transverse beta function entering the wedge, $L_{R}$ is the material radiation length, $\Delta w$ is the length of the absorber at central energy loss, $\mathrm{mc}^{2}=105.66 \mathrm{MeV}$, and $\beta, \gamma$ are the relativistic kinematic factors $(\beta \sim 1, \gamma \sim 29.3)$. With the energy loss at central momentum at $31.5 \mathrm{MeV}$ and $\beta_{t}=2 \mathrm{~m}, \delta \varepsilon=\delta \varepsilon_{N} / \beta \gamma=\{5.0$, $4.4,5.2,6.0\} \times 10^{-6} \mathrm{~m}$ for poly, $\mathrm{Be}, \mathrm{B}_{4} \mathrm{C}, \mathrm{C}$, respectively. This is not too great an increase. But at $\beta_{t}=7 \mathrm{~m}$, the effect is 3.5 times larger. In the reference example $\left(\beta_{y}=7 \mathrm{~m}\right)$, the veritical emittance would be more than doubled, and probably lead to some beam loss in the downstream optics. Changing the optics to smaller $\beta_{y}$ would be very desirable.

Higher-Z materials would have much larger multiple scattering, and would therefore be undesirable.

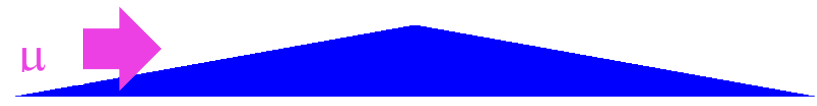

Fig 3. Schematic view of a muon beam incident on a large angle $\left(160^{\circ}\right)$ absorber.

Another important effect is energy straggling, which is the rms energy spread increase from passing through a material, and is approximated by:

$$
\frac{d\left(\Delta E_{r m s}^{2}\right)}{d s} \cong 4 \pi\left(r_{e} m_{e} c^{2}\right)^{2} n_{e} \gamma^{2}\left(1-\frac{\beta^{2}}{2}\right)
$$

where $n_{e}$ is the electron density in the material. This can be rewritten as:

$$
\frac{d\left(\Delta E_{r m s}{ }^{2}\right)}{d s} \cong 0.157 \rho \frac{Z}{A} \gamma^{2}\left(1-\frac{\beta^{2}}{2}\right) \quad(\mathrm{MeV})^{2} / \mathrm{cm}
$$

where $\rho$ is the material density in $\mathrm{gm} / \mathrm{cm}^{3}$. This effect is relatively large for $\mathrm{g}-2$, because of the $\gamma^{2}$ dependence. Following Vavilov, as presented by Leo,[12] this factor should be reduced for muons by a factor of $(1+2 \gamma$ $\left.\left(\mathrm{m}_{\mathrm{e}} / \mathrm{m}_{\mu}\right)+\left(\mathrm{m}_{\mathrm{e}} / \mathrm{m}_{\mu}\right)^{2}\right)$, which is a factor of $\sim 1.3$ for 3.1 $\mathrm{GeV} / \mathrm{c} \mu$ 's, from finite mass effects.

For the example with $28.1 \mathrm{MeV} / \mathrm{c}$ energy loss we obtain $\left(\Delta E_{r m s}\right)^{2}=\{347,325,350,345\} \mathrm{MeV}^{2}$ for poly, $\mathrm{Be}, \mathrm{B}_{4} \mathrm{C}$ and diamond, respectively, and is thus almost material independent.

This leads to an increase in $\delta^{2}$ by $36 \times 10^{-6}$ at the reference. In the baseline example, we would expect this to be added to the cooled $\delta^{2}$ of $\sim 41 \times 10^{-6}$ and compared to the initial value of $144 \times 10^{-6}$. The final $\delta$ would be $\sim 0.0088$, and the increase in $\mathrm{g}-2$ beam would be reduced to $\sim 37 \%$; more than half the possible increase (with ideal exchange) is lost from the energy straggling.

Straggling and multiple scattering can be reduced by choosing a shorter absorber than the reference case. Choosing $\delta^{\prime}$ at 0.6 of the reference value (half angle and resulting width) reduces the multiple scattering and straggling effects by a factor of $\sim 1.7$. The final enhancement in density is about the same. ( $\delta$ is reduced from $1.2 \%$ to $0.74 \%$ without straggling and to $\sim 0.89 \%$ with straggling $(\sim 35 \%$ improvement). The dispersion and betatron function changes are much less $\left(\beta_{\mathrm{x}}: 2 \rightarrow 3.2 \mathrm{~m}\right.$. $\eta: 0.65 \rightarrow 0.31 \mathrm{~m})$. An optimum with $\sim 40 \%$ improvement exists at $\sim 0.8$ the reference value.

The shorter wedges would also have better transverse acceptance (from reduced transverse emittance increase), and would be preferred.

\section{DISCUSSION}

The linear optics optimum is probably not a global optimum for the procedure, particularly after considering straggling and multiple scattering, which may significantly reduce acceptance.

The transverse and longitudinal acceptances of the ring are limited by the ring aperture, which has an $\mathrm{r}=4.5$ $\mathrm{cm}$ radius and the betatron functions $\left(\beta_{x}=8 \mathrm{~m}, \beta_{y}=\right.$ $18.4 \mathrm{~m}, \eta=8.2 \mathrm{~m})$. These are given by: $A_{x}=r^{2} / \beta_{x}$ and $A_{y}=$ $r^{2} / \beta_{x}$ which is $0.00025 \mathrm{~m}$ for $A_{x}$ and $0.00011 \mathrm{~m}$ for $A_{y}$. This could be rewritten as an rms emittance acceptance by dividing by $6\left(\varepsilon_{r m s}=\mathrm{A} / 6\right)$, obtaining $\varepsilon_{x}=0.000042 \mathrm{~m}$, $\varepsilon_{y}=0.000018 \mathrm{~m}$. The transverse emittance increase induced by the absorber should be significantly less than these aperture cuts. (Further aperture cuts are imposed by the injection optics.) This would be true for $\varepsilon_{x}$ but not $\varepsilon_{y}$ in the reference case, because of the larger $\beta_{y}$ at the wedge and in the g- 2 ring. Smaller $\beta_{y}$ at the wedge would be helpful.

Energy straggling increases as $\gamma^{2}$, and it severely limits the application to the $\mathrm{g}-2$ beam because it has a relatively high energy. Lower-energy $\mu$ beams would be less vulnerable to this effect.

The beam optics is changed at the wedge, both in the betatron functions and dispersion, as well as the downstream beam sizes and emittances. For example, at the "optimum" $\delta^{\prime}, \eta=0$ after the wedge; the original g-2 line matches $\eta=0.65$ at the wedge to $\eta=\sim 0$ at the g- 2 ring. The optics should be rematched to an optimum injection $\eta$. $\beta_{y}$ also decreases at the wedge, from the increase in the vertical emittance from multiple scattering, while the vertical beam size is unchanged.

\section{SIMULATION RESULTS}

We have initiated simulations of the g-2 case.[7] In these simulations, beam was tracked using G4Beamline through the transport from the delivery ring into the g-2 ring. Wedges of various materials and dimensions were 
inserted in the high dispersion region and optimized for providing the most beam within $\mathrm{a} \pm 0.1 \% \delta \mathrm{p} / \mathrm{p}$ acceptance window through the transport.

As may be expected from the above discussion, the optimum wedge is a low-Z material (poly $\left(\sim \mathrm{C}_{2} \mathrm{H}_{4}\right)$ or $\mathrm{Be}$ ) with a shallow effective angle $\left(\sim 150-160^{\circ}\right)$, and increases the beam within the acceptance by $\sim 30 \%$. (see fig. 4 ) The wedge increases transverse emittance, and with the mismatched optics the larger amplitude particles were lost in the transport; the $30 \%$ net improvement included the losses.

The result is considered to be enough of an improvement to encourage further development, including further simulation and design and construction of moveable physical wedge inserts in the g-2 transport line at the high dispersion point.

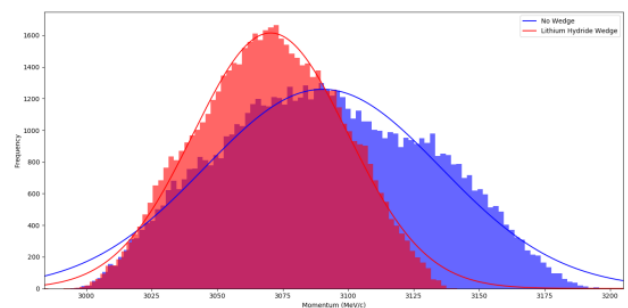

Figure 4. Momentum distribution of beam reaching the g-2 ring without (blue) and with (orange) a wedge. While total number of muons is reduced, the momentum width is reduced and beam within $\pm 0.1 \%$ is increased by $\sim 30 \%$.

In the initial evaluations the beam optics was not rematched following the wedge, and a full simulation with matching into the g-2 ring and storage has not yet been completed. This must be done in the near future. Proper matching could increase acceptance significantly. A modified optics with larger dispersion at the wedge could also greatly improve acceptance.

\section{ACKNOWLEDGEMENT}

We thank M. Syphers for helpful and encouraging discussions.

\section{REFERENCES}

[1] Bartoszek, L. et al., Mu2e Technical Design Report, Mu2e Doc 4299, arXiv 1501.0524.(2015).

[2] Mu2e Conceptual Design Report, Mu2e Document 1169v12 (2012).

[3] Polly, C. et al., Muon g-2 Technical Design Report, Fermilab-FN-0992-E (2014).

[4] D. Neuffer, "Principles and Applications of Muon Cooling", Particle Accelerators 14, 75 (1983).

[5] Neuffer, D., Sayed, H., Acosta, J., Hart, T., and Summers, D., "Final Cooling for a High-Energy High-Luminosity Lepton Collider, 2017_JINST_12_T07003, Journal of Instrumentation 12, T07003 (2017).

[6] Neuffer, D., "Phase Space Exchange in Thick Wedge Absorbers", Fermilab Note MuCOOL-003 (1996), reprinted in Fermilab-FN-1046-AD-APC (2017).

[7] D. V. Neuffer, J. Bradley and D. Stratakis, "Muon Intensity Increase by Wedge Absorbers for low-E Muon Experiments", Proc. COOL2017, Bonn, Germany, September 2017, TUP04, p. 33 (2017).

[8] P. Gruber, "Material Constants for Muon Cooling Simulations", CERN/NUFACT Note 23 (2000).

[9] T. Mohayai, et al.,"A Wedge Test in MICE”, FERMILABCONF-18-234-APC, Proc. IPAC2018 Vancouver, CA (2018).

[10]D. Stratakis et al., "Accelerator performance analysis of the Fermilab Muon Campus", Phys. Rev. Accel. And Beams. 20, 111003 (2017).

[11] D. Stratakis et al., "Commissioning and first results of the Fermilab Muon Campus", Phys. Rev. Accel. And Beams. 20, 011001 (2019).

[12] W. Leo, Techniques for Nuclear and Particle Physics Experiments, Springer-Verlag (1987). 